\title{
Influence of Near-Field Electromagnetic Interactions on Optical Properties of Perfect Lens Consisting of Left-Handed Material
}

\author{
Kuo Pin Chiu and Din Ping Tsai, Senior Member, IEEE
}

\begin{abstract}
In this paper, we investigate the surface plasma resonance of a slab of left-handed material (LHM). We use the transfer matrix method to calculate the attenuated total reflection spectra of the LHM slab. The intrinsic parameters of the LHM slab are suitable chose so that the slab has both negative permittivity and permeability in a frequency range of visible light. We have calculated the cases with different slab thickness and various dielectrics surrounding the LHM slab.
\end{abstract}

Index Terms-Attenuated total reflection (ATR) spectrum, lefthanded material (LHM), nanoslab, surface plasmons.

\section{INTRODUCTION}

$\mathbf{T}$ HE electromagnetic (EM) radiation emitted or scattered by an object consists of radiative component of propagating modes and near-field component of evanescent modes. The evanescent modes are the high-frequency Fourier field components describing the rapidly varying spatial features on the object. Because of losing these evanescent fields in the far-field imaging, the subwavelength features of the source cannot be resolved. This limitation on spatial resolution of conventional farfield optical system is called diffraction limit. Recently, Pendry [1] proposed a concept of superlens which is capable of focusing all Fourier components of the source onto a two-dimensional image with a resolution far below the diffraction limit. This property of left-handed material (LHM) can be applied to optical data storage to increase the resolution of recording marks or to reduce the spot size of light. On the other hand, some investigations [2], [3] have shown that the properties of amplifying evanescent waves and subwavelength imaging in a lossy LHM slab are closely related to the resonance of SP modes at the two surfaces of the LHM slab. In this paper, we will investigate the interactions between light and surface plasmons (SPs) of a nanoslab which is assumed to be LHM in the visible frequency region.

\section{BASIC PRINCIPLE OF CALCUlation}

SPs are collective oscillations of surface charge density that propagate in a wavelike manner at the interface between two

Manuscript received August 27, 2004. This work was supported in part by the National Science Council, Taiwan, R.O.C. under Grant NSC-93-2112-M002-007, and the Ministry of Economic Affairs, Taiwan, R.O.C., under Grant 93-EC-17-A-08-S1-0006.

The authors are with the Department of Physics, National Taiwan University, Taipei 10617, Taiwan, R.O.C., and also with the Center of Nanostorage Research, National Taiwan University, Taipei 10617, Taiwan, R.O.C. (e-mail: kpchiu@ntu.edu.tw; dptsai@phys.ntu.edu.tw).

Digital Object Identifier 10.1109/TMAG.2004.842046 media. The EM fields of SPs can propagate along the interface but decay exponentially away from it. For a dispersive thin slab, with thickness $d$, SP modes can exist simultaneously on both interfaces of the slab. If the slab is very thin, these modes will couple to form two coupled-mode solutions. Based on the evanescent behavior of the SP fields and assuming that the dielectrics surrounding the slab are the same, the dispersion relations of SPs can be written as the following form [4]:

$$
\begin{aligned}
\varepsilon_{(\omega)} & =-\varepsilon_{m}\left(\frac{k_{z}}{k_{m z}}\right) \operatorname{coth}\left(\frac{k_{z} d}{2}\right) \\
\varepsilon_{(\omega)} & =-\varepsilon_{m}\left(\frac{k_{z}}{k_{m z}}\right) \tanh \left(\frac{k_{z} d}{2}\right) \\
\mu_{(\omega)} & =-\mu_{m}\left(\frac{k_{z}}{k_{m z}}\right) \operatorname{coth}\left(\frac{k_{z} d}{2}\right) \\
\mu_{(\omega)} & =-\mu_{m}\left(\frac{k_{z}}{k_{m z}}\right) \tanh \left(\frac{k_{z} d}{2}\right) .
\end{aligned}
$$

Equations (1) and (2) are the dispersion relations for p-and s-polarized incident fields, respectively. $\varepsilon_{(\omega)}, \mu_{(\omega)}$ and $\varepsilon_{m}, \mu_{m}$ are the permittivity and permeability of the slab and its surrounding dielectrics, respectively. $k_{z}$ and $k_{m z}$ represent the normal components of the wave vector in the slab and the surrounding dielectric, respectively. These dispersion relations describe the existed SP modes of the system. SPs can be excited when the frequency and wave vector of the incident light matches the points on the dispersion curves.

The effective permittivity and permeability of a LHM can be expressed as [5], [6]

$$
\begin{aligned}
& \varepsilon_{(\omega)}=1-\frac{\omega_{p}^{2}}{\left(\omega^{2}+i \omega \gamma\right)} \\
& \mu_{(\omega)}=1-\frac{F \omega^{2}}{\left(\omega^{2}-\omega_{0}^{2}+i \omega \Gamma\right)}
\end{aligned}
$$

where $\omega$ is the frequency of the incident light, $\omega_{p}$ is the effective plasma frequency, $F$ is a volume factor value, and $\omega_{0}$ is the magnetic resonance frequency. The damping terms $\gamma$ and $\Gamma$ represent dissipation of EM energy into the system. The parameters described above are all dependent on the constitutive structures of the LHM. By scaling down the dimensions of the building blocks of the LHM, it is possible to fabricate a LHM that operates at optical and infrared frequencies [7]. The dispersion curves of a nanoslab consisting of LHM can be calculated according to (3) together with (1) and (2). 
In our calculations, the transfer matrix method is used to calculate the ATR spectra. Consider a p-polarized wave incident through a layered system with an angle $\theta$. The field components $H_{x(z)}$ and $E_{y(z)}$ at any $z=$ constant plane can be described by the following relation:

$\left[\begin{array}{c}H_{x(0)} \\ E_{y(0)}\end{array}\right]=M_{(z)} \cdot\left[\begin{array}{c}H_{x(z)} \\ E_{y(z)}\end{array}\right]$ with $M_{(z)}=M_{1\left(d_{1}\right)} \cdot M_{2\left(d_{2}\right)} \cdot M_{3\left(d_{3}\right)} \cdots$

where $H_{x(0)}$ and $E_{y(0)}$ are the $x$-and $y$-components of magnetic and electric fields at the $z=0$ plane, that is, the plane at which wave field is incident. The transfer matrix $M_{j(d j)}$ has the form of

$M_{j\left(d_{j}\right)}=\left[\begin{array}{cc}\cos \left(k_{z j} d_{j}\right) & -\left(\frac{i}{p_{j}}\right) \sin \left(k_{z j} d_{j}\right) \\ -i p_{j} \sin \left(k_{z j} d_{j}\right) & \cos \left(k_{z j} d_{j}\right)\end{array}\right], j=1,2, \ldots$

In (5), $d_{j}$ and $\varepsilon_{j}$ are the thickness and permittivity of the $j$ th layer and $p_{j}=(c / \omega)\left(k_{z j} / \varepsilon_{j}\right) . k_{z j}$ is the $z$-component of wave vector in the $j$ th layer. The constant $c$ is speed of light in vacuum. The $M_{j(d j)}$ for s-polarized wave has the same form as (5), but just replacing the quantity $\varepsilon_{j}$ by $\mu_{j}$ in $p_{j}$ of the associated layer. In our calculations, the elements of the transfer matrix consist of $\varepsilon_{j}, \mu_{j}$, and $k_{z j}$ associated with each layer instead of the refractive index and angle. With this form of representation, it is more convenient to describe the propagation of EM wave through a LHM which has both dispersive permittivity and permeability.

\section{RESULTS AND DISCUSSIONS}

We have calculated the ATR spectra for a nanoslab consisting of LHM. The parameters of the LHM slab used in our calculations are described as follows. The value of $\omega_{p}$ in (3) is $3570 \mathrm{THz}$. Both the damping terms $\gamma$ and $\Gamma$ of LHM are set to be $19.4 \mathrm{THz}$. When the light frequency is smaller than $\omega_{p}$, the permittivity of the slab is negative. The other parameters used in (3) are: $F=0.56, \omega_{0}=3142 \mathrm{THz}$ (corresponding to $600 \mathrm{~nm}$ wavelength). It can be shown that the permeability of the slab is negative in the frequency range between $3142 \mathrm{THz}$ (i.e., $\omega_{0}$ ) and $4736 \mathrm{THz}$ (it is defined as $\omega_{m p}$ ). It is seen that under these parameters, the LHM slab has both negative permittivity and permeability in the frequency range between $\omega_{0}$ and $\omega_{p}$, i.e., within the visible frequency range. The configuration for calculating the ATR spectra is shown in Fig. 1. The permittivity and permeability of the prism are set to be 3 and 1 , respectively. The incident angle, $\theta$, of light is $75^{\circ}$. The separation between the prism and the slab of LHM $a$ is $100 \mathrm{~nm}$.

We have calculated the ATR spectra for cases with different thickness of the LHM slab. The minimum peaks in ATR spectrum result from the resonances of SP modes. The variation of ATR resonant frequencies for p-polarized modes with increasing slab thickness is shown in Fig. 2(a). The modes denoted as p-1 and p-2 occur in the frequency region $\omega<\omega_{0}$. In this region, $\mu_{(\omega)}$ is positive and $\varepsilon_{(\omega)}$ is negative. The slab behaves similar to conventional metal in this region. It is seen that the separation between $\mathrm{p}-1$ and $\mathrm{p}-2$ resonant peaks decreases when the thickness of the LHM slab increases. This is an expected result because of that the separation of $\mathrm{p}-1$ and $\mathrm{p}-2$ modes comes from
Dielectric 2

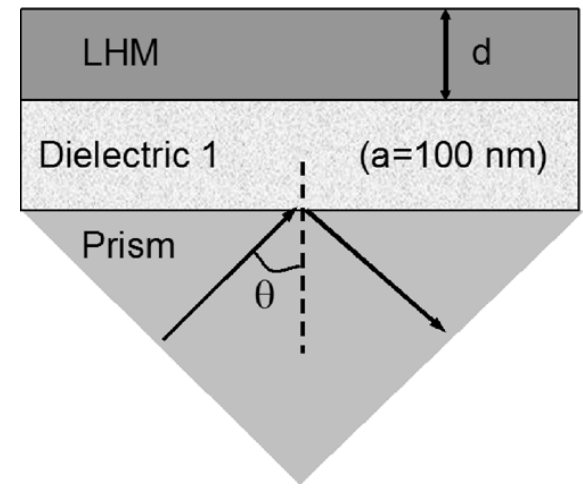

Fig. 1. Configuration used for calculating ATR spectrum.

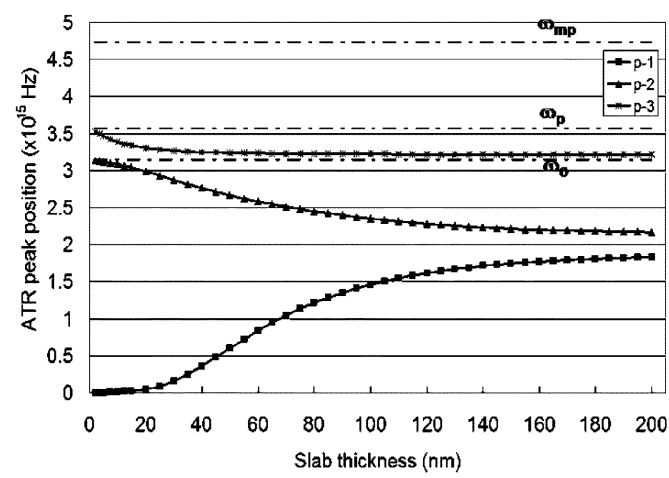

(a)

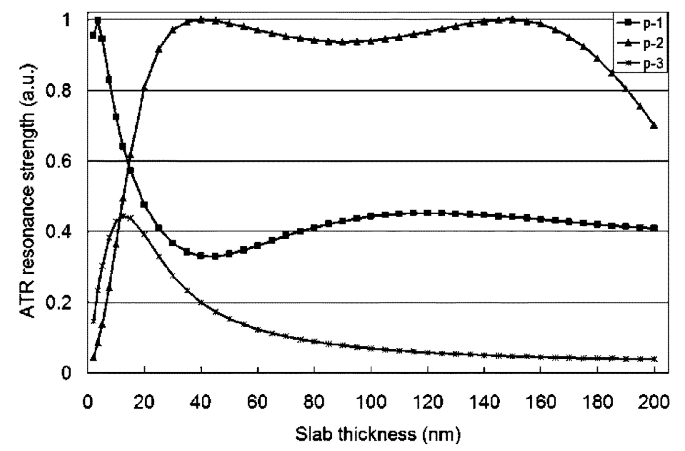

(b)

Fig. 2. (a) ATR resonant frequency, for p-polarized modes, versus the thickness of the LHM slab. (b) Variation of resonant strength with the thickness of the slab for each ATR peaks corresponding to (a).

the coupling of the SPs at the two interfaces of the LHM and when the thickness of the slab increases the coupling effect will be reduced, so that the separation decreases. Fig. 2(b) shows the variation of resonant strength with increasing thickness of the slab for each ATR peaks corresponding to Fig. 2(a). The resonant strength of the ATR spectrum is taken to be $1-R$, where $R$ represents the reflectivity. We can see that the resonant strength of p-1 mode decreases rapidly with increasing thickness of the slab. For p-2 mode, the resonant strength is much stronger then the p-1 mode when the thickness of slab increases. It seems that the decreasing resonant strength of $\mathrm{p}-1$ mode is accompanied by the increasing resonant strength of $\mathrm{p}-2$ mode. On the other hand, there are two maximum values in the curve of the resonant strength for both the p-1 and p-2 modes with increasing thickness of the slab. 


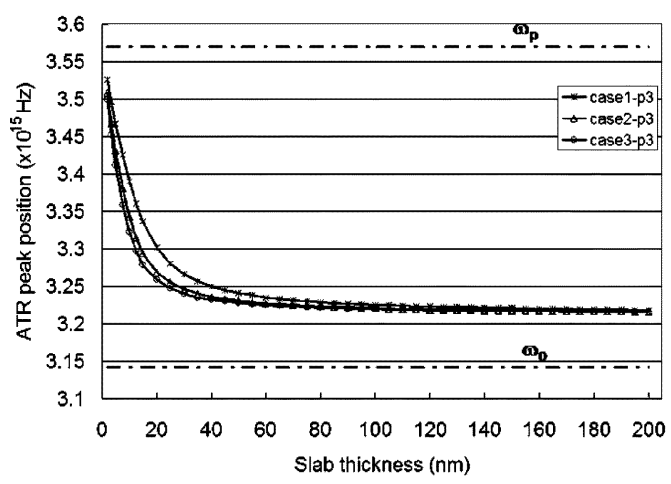

(a)

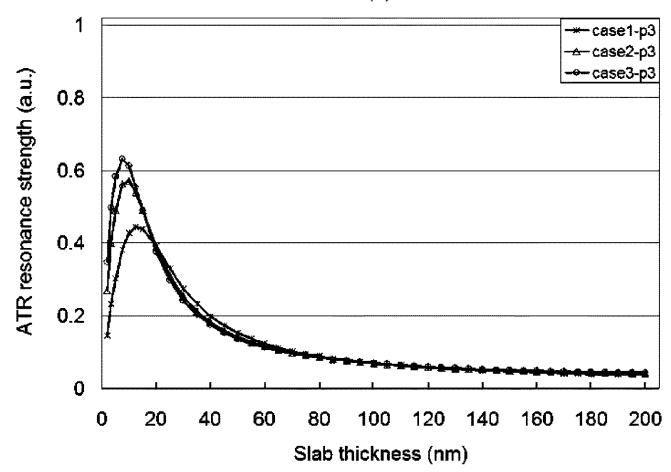

(b)

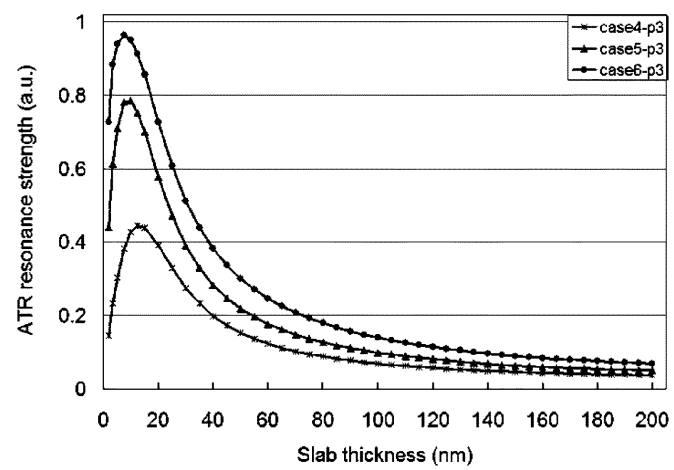

(c)

Fig. 3. (a) Variation of ATR resonant frequency with slab thickness for $\mathrm{p}-3$ mode in different cases. (b) and (c) Variation of ATR resonant strength with slab thickness for p-3 mode in different cases.

The mode denoted as p-3 is also p-polarized mode, and it always falls in the frequency range where the slab material has both negative permittivity and permeability, as seen in Fig. 2(a). It would play the most important role that is closely related to the perfect imaging property of LHM slab. In Fig. 2(b) we see that with increasing thickness of the LHM slab, the resonant strength of p-3 mode increases to a maximum value and then decreases progressively. We show some more calculated results of the p-3 SP mode in Fig. 3. These calculated results are corresponding to cases that the dielectrics surrounding the LHM slab are changed. For the cases 1, 2, and 3, the dielectric 1 in Fig. 1 is set to be air and replacing the dielectric 2 by air, water, and glass, respectively. For the cases 4, 5, and 6, the dielectric 2 in Fig. 1 is set to be air and replacing the dielectric 2 by air, water, and glass, respectively.

Fig. 3(a) shows the variation of resonant peak position with increasing thickness of the LHM slab for the cases 1 to 3 . It is found that the cases 4 to 6 have the same results as Fig. 3(a). This is a reasonable result because the SP modes are determined by the kinds of materials that form the interface. In our calculated cases, for example, 2 and 5, they have the same coupled SP modes at the two surfaces of the LHM slab so that the resonant positions are the same. It is interesting that when the permittivity of dielectric 2 increases, the maximum resonant strength occurs at smaller thickness of the LHM slab and the maximum strength becomes stronger, as shown in Fig. 3(b). Similar phenomena have been seen in Fig. 3(c) which is associated to the cases of increasing the permittivity of dielectric 1. Comparing Fig. 3(b) and (c), it is found that the maximum resonant strength of the p-3 SP mode grows more rapidly by increasing the permittivity of dielectric 1 than by increasing the permittivity of dielectric 2 .

\section{SUMMARY}

The SP modes of a LHM slab in visible frequency region have been calculated with proper parameters. Our calculated results demonstrate that the SP resonances have maximum strength at some special slab thickness of the LHM. And different SP resonant modes have different response to the variation of slab thickness. The resonant strength and coupling behavior of the SPs are also influenced by the thickness of the LHM and its surrounding dielectrics. Because the light sources used in optical disks are visible light, these results will be helpful to us for choosing structure parameters when applying LHM slab to optical disks. We can modify the behaviors of the SP resonances and consequently the optical response of the LHM slab by varying the constitutive structure or surrounding material of the slab.

\section{REFERENCES}

[1] J. B. Pendry, "Negative refraction makes a perfect lens," Phys. Rev. Lett., vol. 85, pp. 3966-3969, 2000.

[2] X. S. Rao and C. K. Ong, "Amplification of evanescent waves in a lossy left-handed material slab," Phys. Rev. B, Condens. Matter, vol. 68, p. $113103,2003$.

[3] — "Subwavelength imaging by a left-handed material superlens," Phys. Rev. E, Stat. Phys. Plasmas Fluids Relat. Interdiscip. Top., vol. 68, p. $067601,2003$.

[4] R. Ruppin, "Surface polaritons of a left-handed material slab," J. Phys. Condens. Matter, vol. 13, pp. 1811-1819, 2001.

[5] J. B. Pendry, A. J. Holden, W. J. Stewart, and I. Youngs, "Extremely low frequency plasmons in metallic mesostructures," Phys. Rev. Lett., vol. 76, pp. 4773-4776, 1996

[6] J. B. Pendry, A. J. Holden, D. J. Robbins, and W. J. Stewart, "Magnetism from conductors and enhanced nonlinear phenomena," IEEE Trans. Microwave Theory Tech., vol. 47, no. 11, pp. 2075-2084, Nov. 1999.

[7] N. C. Panoiu and R. M. Osgood, Jr., "Numerical investigation of negative refractive index metamaterials at infrared and optical frequencies," Opt. Commun., vol. 223, pp. 331-337, 2003. 